\title{
PAPER
}

\section{An extension of Poincaré group based on generalized Fermi-Walker coordinates}

To cite this article: Josep Llosa 2017 Class. Quantum Grav. 34205003

\section{Manuscript version: Accepted Manuscript}

Accepted Manuscript is "the version of the article accepted for publication including all changes made as a result of the peer review process, and which may also include the addition to the article by IOP Publishing of a header, an article ID, a cover sheet and/or an 'Accepted

Manuscript' watermark, but excluding any other editing, typesetting or other changes made by IOP Publishing and/or its licensors"

This Accepted Manuscript is (c) (c) 2017 IOP Publishing Ltd.

During the embargo period (the 12 month period from the publication of the Version of Record of this article), the Accepted Manuscript is fully protected by copyright and cannot be reused or reposted elsewhere.

As the Version of Record of this article is going to be / has been published on a subscription basis, this Accepted Manuscript is available for reuse under a CC BY-NC-ND 3.0 licence after the 12 month embargo period.

After the embargo period, everyone is permitted to use copy and redistribute this article for non-commercial purposes only, provided that they adhere to all the terms of the licence https://creativecommons.org/licences/by-nc-nd/3.0

Although reasonable endeavours have been taken to obtain all necessary permissions from third parties to include their copyrighted content within this article, their full citation and copyright line may not be present in this Accepted Manuscript version. Before using any content from this article, please refer to the Version of Record on IOPscience once published for full citation and copyright details, as permissions will likely be required. All third party content is fully copyright protected, unless specifically stated otherwise in the figure caption in the Version of Record.

View the article online for updates and enhancements. 


\title{
An extension of Poincaré group based on generalized Fermi-Walker coordinates

\author{
Josep Llosa \\ Departament de Física Quàntica i Astrofísica, Institut de Ciències del Cosmos (ICCUB), \\ Universitat de Barcelona, Martí Franquès 1, E08028 Barcelona, Spain
}

August 25, 2017

\begin{abstract}
The class of accelerated and rotating reference frames has been studied on the basis of generalized Fermi-Walker coordinates. We obtain the infinitesimal transformations connecting any two of these frames and also their commutation relations. We thus have an infinite dimensional extension of the Poincare algebra and, although it turns out to be Abelian extension, and hence trivial, it is noteworthy that, contrarily to Lorentz boosts, acceleration and rotational boost generators commute with each other and with the generators of Poincaré group as well.

PACS number: 02.40.Ky, 02.20.Tw, 02.20.Sv, 04:20.Cv,
\end{abstract}

\section{Introduction}

The laws of Newtonian mechanics hold in all inertial reference frames, which are in uniform rectilinear motion with respect to each other. This is known as the principle of relativity of Galilei. However, this principle of relativity can be extended to arbitrary rigid frames, which are in arbitrary translational and rotational motion with respect to each other, i. e. they are mutually related by 
ExtendedPrinciple6 v0.tex

coordinate transformations like

$$
x^{i}=R_{j}^{i}(t) x^{j}+s^{i}(t), \quad t^{\prime}=t+t_{0},
$$

where $R_{j}^{i}(t)$ is an orthogonal matrix and $s^{i}(t)$ arbitrary functions of time. The laws of Newtonian mechanics have the same form in any of these coordinate systems, provided that the necessary inertial force fields — dragging, Coriolis, centrifugal, ... - are included.

According to the special theory of relativity the laws of physics hold in all Lorentzian reference frames, the relative motion of any couple of these frames is rectilinear and uniform, and the coordinates in any pair of these frames are connected by a Poincaré transformation. Endeavouring to set up a theory of gravity consistent with his theory of relativity, Einstein initially aimed to generalize the theory of relativity to accelerated motions [1], but he soon abandoned this idea in favour of the principle of general covariance. Its invariance group, namely spacetime diffeomorphisms, is much wider than Poincaré group but, as soon Kretschmann pointed out [2], [3] "since any theory, whatever its physical content, can be rewritten in a generally covariant form, the group of general coordinate transformations is physically irrelevant" [4]. Moreover, in Kretschmann's view, special relativity is the one with the relativity postulate of largest content; indeed, its isometry group is a ten-parameter group, which is the largest isometry group in four dimensions, whereas for generic spacetimes in general relativity the isometry group reduces to the identity.

More recently other authors have insisted in the convenience of restricting general covariance [5] and even a Principle of restricted covariance has been explicitly stated [6]. There is also in the literature a renewed interest in accelerated reference frames. In a non-relativistic approach we should mention the extensions of Galilei algebra to encompass acceleration in the context of non-relativistic quantum mechanics [7], [8], [9] and, from a relativistic standpoint, the approach by Mashhoon [10] in view of its possible application to non-local gravitational theories [11].

Our aim is to find an extension of the principle of relativity, meaning that there is a class of coordinate systems - larger that the Lorentzian class but more restrictive than general curvilinear coordinates- such that the laws of physics have the same form, including inertial force fields if necessary; in the same way as the extended principle of relativity for rigid reference frames holds in 
Newtonian mechanics, as commented at the start.

Fermi-Walker (FW) coordinates [12], [13] are characterized by an arbitrary origin worldline, whose proper velocity stands for the time axis, whereas the triad of space axis evolve without rotating in a specific way, namely Fermi-Walker transport [14], just to stay orthogonal to proper velocity. Often these coordinates have been seen as the relativistic generalization of the coordinates associated to an accelerated, non-rotating observer [12], [15], [16]. From a historical viewpoint, FW coordinates have also the merit of coinciding (for rectilinear motion) with the coordinate systems considered by Einstein in his pioneering work [1], as has been proved elsewhere[17].

This class of observers and associated coordinates can be extended by allowing the space axes to have an arbitrary rotational motion [12], [18], [19]. We shall refer to these as generalized FermiWalker coordinates $(\mathrm{GFW})^{1}$. Particularly ref. [18] aims to distinguish the inertial and gravitational effects in the proper reference frame of an accelerated rotating observer.

We have concentrated on GFW coordinates because several authors have chosen them as the most suitable to describe relativistic physical situations in which an extended non-inertial laboratory apparatus intervenes ${ }^{2}$, e. g. at rest on the Earth surface. To list a few: the energy-momentum tensor for a Casimir apparatus in a weak gravitational field [20], the Fulling-Unruh effect in accelerated frames [21], the gravitational perturbations of the hydrogen spectrum [22], [23], and also [24], [25], $[26]$.

In section 2 we outline the main features of FW and GFW coordinate systems in Minkowski spacetime and see the specific form of Minkowski interval when written in these coordinates. Then in section 3 we prove that the latter specific form is exclusive of GFW coordinates and state a kind of uniqueness result associated to them.

The Minkowski metric components in these coordinates are ten well defined functions, namely $g_{\mu \nu}\left(X^{j}, T ; f_{J}(T)\right)$ that involve six functions of time $f_{J}(T), J=1 \ldots 6$. These functions are connected with the proper acceleration of the origin and the angular velocity of the spatial triad of

\footnotetext{
${ }^{1}$ Gourgoulhon [19] uses the term "coordinates with respect to a generic observer"

${ }^{2}$ Alternatively, FW coordinates if there is no rotational motion or Fermi normal coordinates for a freely falling observer in a gravitational field
} 
axes.

In passing from one GFW system of coordinates to another the $g_{\mu \nu}\left(X^{j}, T ; f_{J}\right)$ are form invariant, i.e. considered as functions of the ten variables $X^{j}, T$ and $f_{J}$ they do not change. However they are not isometries in the proper sense because, if we consider the components as functions of the four coordinates, namely $\bar{g}_{\mu \nu}\left(X^{j}, T\right):=g_{\mu \nu}\left(X^{j}, T ; f_{J}(T)\right)$, their values do change because the six functions $f_{J}(T)$ differ from one GFW system to the other. This dual feature induce us to use the term generalized isometries [29].

We then study the infinitesimal transformations connecting two GFW coordinate systems, as the solutions of a generalized Killing equation, and derive the infinitesimal generators . As these transformations imply not only a change in the spacetime coordinates $\left(X^{j}, T\right)$ but also in the six functions $f_{J}(T)$ characterizing the GFW system, the infinitesimal generators act on a manifold that is much larger than merely Minkowski spacetime (an infinite dimensional one, actually). These generators span an infinite dimensional extension of Poincaré algebra which includes acceleration and rotation, which may be taken as the mathematical embodying of an extension of the principle of special relativity abiding arbitrary translational and rotational motions.

\section{Generalized Fermi-Walker coordinates}

Let $z^{\mu}(\tau)$ be a timelike worldline in ordinary Minkowski spacetime, and $u^{\mu}=\dot{z}^{\mu}(\tau)$ and and $a^{\mu}=\ddot{z}(\tau)$ the proper velocity and acceleration 4-vectors. (We take $c=1$, Greek indices run from 1 to 4 and Latin indices from 1 to $3 ; x^{\mu}$ refer to Lorentzian coordinates and the summation convention is always understood unless the contrary is explicitely said.)

A 4-vector $w^{\mu}(\tau)$ is Fermi-Walker transported [13] along $z^{\mu}(\tau)$ if

$$
\frac{\mathrm{d} w^{\mu}}{\mathrm{d} \tau}=\left(u^{\mu} a_{\nu}-u_{\nu} a^{\mu}\right) w^{\nu}
$$

Let us now consider an orthonormal tetrad, $\hat{e}_{(\alpha)}^{\mu}(\tau)$, which is FW transported along $z^{\mu}(\tau)$ and such that $\hat{e}_{(4)}^{\mu}=u^{\mu}$. For a given point in spacetime, $x^{\mu}$, the Fermi-Walker coordinates [13], [19] with space origin on $z^{\mu}(\tau)$ are: 
The time $T\left(x^{\nu}\right)$, given as an implicit function by

$$
\left[x^{\mu}-z^{\mu}(T)\right] u_{\mu}(T)=0
$$

The space coordinates $X^{i}$, defined by

$$
\hat{X}^{i}=\left[x_{\mu}-z_{\mu}(T(x))\right] \hat{e}_{(i)}^{\mu}(T(x))
$$

FW coordinates are the local coordinates of a non-rotating accelerated observer [12]. A natural generalization, that also includes arbitrary rotational motion, is based on the notion of generalized Fermi-Walker (GFW) transport [12], [18] of a vector $w^{\mu}$ along the worldline $z^{\mu}(\tau)$ :

$$
\frac{\mathrm{d} w^{\mu}}{\mathrm{d} \tau}=\Omega_{\nu}^{\mu}(\tau) w^{\nu}
$$

where

$$
\Omega_{\nu}^{\mu}=u^{\mu} a_{\nu}-a^{\mu} u_{\nu}-\epsilon_{\nu \alpha \beta}^{\mu} \omega^{\alpha} u^{\beta}
$$

is the (spacetime) angular velocity and $\omega^{\alpha}(\tau)$ is an arbitrary vector that is orthogonal to $u^{\mu}$ that we shall call proper angular velocity 4 -vector.

Consider now a new tetrad $\left\{e_{(\alpha)}^{\nu}(\tau)\right\}_{\alpha=1 \ldots 4}$, with $e_{(4)}^{\nu}(\tau)=u^{\nu}(\tau)$, that is GFW transported along $z^{\mu}(\tau)$. We shall write:

$$
e_{\nu(\alpha)}=\eta_{\nu \mu} e_{(\alpha)}^{\nu}, \quad e^{\nu(\alpha)}=e_{(\beta)}^{\nu} \eta^{\beta \alpha}
$$

and the components of the angular velocity in this comoving base are:

$$
\hat{\Omega}_{\beta}^{\alpha}=\Omega_{\nu}^{\mu} e_{\mu}^{(\alpha)} e_{(\beta)}^{\nu}, \quad \hat{\Omega}_{i}^{4}=\hat{\Omega}_{4}^{i}=\hat{a}^{i}, \quad \hat{\Omega}_{j}^{i}=\epsilon_{j k}^{i} \hat{\omega}^{k}
$$

where $\hat{\omega}^{l}=\omega_{\nu} e_{(l)}^{\nu}$. In a terminology borrowed from rigid body kinematics, we will refer to the GFW transported tetrad $\left\{e_{(\alpha)}^{\nu}(\tau)\right\}_{\alpha=1 \ldots 4}$ as the "body axes", whereas the inertial frame base will be called the "spacetime axes".

On the basis of the origin worldline $z^{\mu}(\tau)$ and the space axes $e_{(i)}^{\mu}$ we can introduce the generalized Fermi-Walker coordinates of a point $x^{\nu}$ : (a) the time $T(x)$ is defined as in equation (2) and (b) the space coordinates are

$$
X^{j}=\left[x_{\mu}-z_{\mu}(T(x))\right] e_{(i)}^{\mu}(T(x))
$$


The inverse coordinate transformation - from GFW to Lorentzian coordinates - is

$$
\left(T, X^{l}\right) \longrightarrow x^{\mu}, \quad x^{\mu}=z^{\mu}(T)+X^{l} e_{(l)}^{\mu}(T)
$$

whence it easily follows that

$$
\mathrm{d} x^{\mu}=\left[(1+\vec{X} \cdot \vec{a}) u^{\mu}+\epsilon_{i k}^{l} \hat{\omega}^{k} X^{i} e_{(l)}^{\mu}\right] \mathrm{d} T+e_{(l)}^{\mu} \mathrm{d} X^{l}
$$

and the Minkowski metric in GFW coordinates is

$$
\mathrm{d} s^{2}=\mathrm{d} \vec{X}^{2}+2 \mathrm{~d} T \mathrm{~d} \vec{X} \cdot(\vec{X} \times \vec{\omega})-\mathrm{d} T^{2}\left[(1+\vec{X} \cdot \vec{a})^{2}-(\vec{X} \times \vec{\omega})^{2}\right]
$$

where the usual standard 3-vector notation has been introduced for the sake of brevity, with $\vec{\omega}=$ $\left(\hat{\omega}^{1}, \hat{\omega}^{2}, \hat{\omega}^{3}\right)$ and $\vec{a}=\left(\hat{a}^{1}, \hat{a}^{2}, \hat{a}^{3}\right)$. This formula generalizes those obtained in refs. [27] and [28] for constant proper acceleration and angular velocity.

For $\hat{\omega}^{i}(t)=\hat{a}^{i}(t)=0$ the interval (10) is Minkowski metric, as expected. Indeed, in this case proper acceleration and angular velocity vanish, the origin worldline is straight, the tetrad is constant and GFW coordinates yield the Lorentzian coordinates for these axes and origin. As a consequence, the group of transformations connecting GFW coordinates ${ }^{3}$ will include Poincaré group as a subgroup. Had we to compare these GFW coordinates with the ordinary FW coordinates based on the same world line, we should obtain $X^{j}=R_{i}^{j}(T) \hat{X}^{i}$, where $R_{i}^{j}(\tau)$ is a rotation matrix satisfying

$$
\dot{R}_{i}^{j}(\tau)=\epsilon_{i k}^{l} \hat{\omega}^{k}(\tau) R_{l}^{j}(\tau)
$$

$\left(\epsilon_{j k}^{l}=\epsilon_{l j k}\right.$ is the three dimensional Levi-Civita symbol, regardless the position of the Latin indices). It is worth to remark that, whereas $a^{\mu}, \omega^{\nu}$ and $\Omega_{\mu \nu}$ are the components of respectively proper acceleration, proper angular velocity vector and spacetime angular velocity on an external Lorentzian coordinate base, $\hat{a}^{j}(\tau), \hat{\omega}^{j}(\tau)$ and $\hat{\Omega}_{\alpha \beta}(\tau)$ are the components of these objects with respect to the GFW transported base. This is why we shall refer to them as intrinsic proper acceleration and so on.

\footnotetext{
${ }^{3}$ The structure of the group of transformations is explained in detail in Appendix D
} 


\subsection{The GFW reference system with origin $z^{\mu}(\tau)$ and angular velocity $\Omega^{\mu}{ }_{\nu}(\tau)$}

Any GFW transported tetrad with angular velocity $\Omega^{\mu}{ }_{\nu}(\tau)$ is a solution of the linear ordinary differential system (4-5). Its general solution is

$$
e_{(\alpha)}^{\mu}(\tau)=\Lambda_{\nu}^{\mu}(\tau) e_{(\alpha)}^{\nu}(0)
$$

where $\Lambda_{\nu}^{\mu}(\tau)$ is a solution of the differential system

$$
\dot{\Lambda}_{\nu}^{\mu}=\Omega_{\rho}^{\mu}(\tau) \Lambda_{\nu}^{\rho}, \quad \text { with } \quad \Lambda_{\nu}^{\mu}(0)=\delta_{\nu}^{\mu}
$$

Therefore two tetrads, $e_{(\alpha)}^{\mu}=e_{(\alpha)}^{\prime \mu}$, that are GFW transported along the same worldline with the same angular velocity will only differ in their initial values and as, besides $e_{(4)}^{\mu}=e_{(4)}^{\prime \mu}=u^{\mu}$, these initial values are connected by a space rotation

$$
e_{(\alpha)}^{\prime \mu}(0)=\sum_{\beta=1}^{4} e_{(\beta)}^{\mu}(0) R_{\alpha}^{\beta}, \quad \text { with } \quad R_{4}^{\beta}=R_{\beta}^{4}=\delta_{4}^{\beta},
$$

$\left(R^{i}{ }_{j}\right)_{i, j=1 \ldots 3}$ being a constant orthogonal matrix. Combining the latter with (11) we have that

$$
e_{(\alpha)}^{\prime \mu}(\tau)=e_{(\beta)}^{\mu}(\tau) R_{\alpha}^{\beta}
$$

Hence all GFW transported tetrads along a given worldline with the same angular velocity are the same apart from an initial space rotation and, according to the definition the GFW coordinates based on any of these tetrads will differ at most in a constant rotation:

$$
\tau^{\prime}=\tau, \quad X^{i}=R_{j}^{i} X^{\prime j}
$$

Given a GFW coordinate system with origin $z^{\mu}(\tau)$ and angular velocity $\Omega_{\alpha}^{\mu}(\tau)$, the worldline $X^{i}=$ constant, $\tau \in \mathbb{R}$ is the "history" of a material point at rest in the GFW reference system. The 3-parameter class of these worldlines (for all $X^{j}$ ) represents the motion of a material body comoving with the GFW system. As commented above, in any other GFW coordinates system based on the same $z^{\mu}(\tau)$ and $\Omega_{\alpha}^{\mu}(\tau)$ we shall still have that $X^{\prime i}=\left(R^{-1}\right)_{j}^{i} X^{j}=$ constant.

The equation defining this 3 -parameter congruence is

$$
\varphi^{\mu}(T, \vec{X})=z^{\mu}(T)+X^{i} e_{(i)}^{\mu}(T), \quad \tau \in \mathbb{R}
$$


and the proper time rate at the place $\vec{X}$ is

$$
\mathrm{d} \tau=\gamma^{-1} \mathrm{~d} T, \quad \gamma:=\left([1+\vec{X} \cdot \vec{a}(\tau)]^{2}-[\vec{X} \times \vec{\omega}]^{2}\right)^{-1 / 2} ;
$$

This is the time ticked by a stationary atomic clock at $\vec{X}$ and it coincides with $T$ at the origin. In general, $\tau \neq T$ and usually the readings of proper time $\tau$ by two stationary clocks at two different places will not keep synchronized. It is thus more convenient to use the synchronous time $T$ instead of local proper time.

The proper velocity vector of the worldline $\vec{X}=$ constant at the synchronous time $T$ is

$$
U^{\mu}(T, \vec{X})=\gamma\left[(1+\vec{X} \cdot \vec{a}) u^{\mu}+\epsilon_{i k}^{l} X^{i} \hat{\omega}^{k} e_{(l)}^{\mu}\right]
$$

The first term corresponds to the origin translational velocity, whereas the second term reflects the rotational motion.

Due to the presence of a square root in the denominator, the domain where the GFW coordinates are valid is restricted to the region $|1+\vec{X} \cdot \vec{a}|>|\vec{X} \times \vec{\omega}|$ and the equality defines the horizon of the GFW coordinate system.

Equations (15) and (16) generalize similar formulae derived in ref. [27]) in the context of constant proper acceleration and angular velocity. Notice that the proper velocity $U^{\mu}$ depends on the place (if $\vec{\omega} \neq 0$, then $U^{\mu}$ is different from the origin proper velocity, unless $\vec{X} \| \vec{\omega}$ ). Proper acceleration also varies from place to place; therefore, we should not speak of "the acceleration of a system of coordinates" but rather the acceleration of the origin, namely $\hat{a}_{i}$ in the body axes.

\section{Uniqueness}

We now prove that the expression (10) of the metric is exclusive for GFW coordinates in Minkowski spacetime.

Proposition 1 If in some coordinate system $\left(X^{i}, T\right)$ the spacetime metric has the form (10), then there is a worldline $z^{\mu}(\tau)$ and an orthonormal tetrad $e_{(\alpha)}^{\mu}(\tau), \alpha=1 \ldots 4$, such that $\left(X^{i}, T\right)$ are the GFW coordinates based on that worldline and tetrad in a locally Minkowskian spacetime. 
Proof: It is straightforward to check that the Riemann tensor for the metric (10) vanishes; therefore the spacetime is locally Minkowskian.

Then consider the matrix $\hat{\Omega}_{\beta}^{\alpha}(\tau)$ defined by

$$
\hat{\Omega}^{i}{ }_{j}(\tau)=\epsilon_{j k}^{i} \hat{\omega}^{k}(\tau), \quad \hat{\Omega}_{i}^{4}(\tau)=\hat{\Omega}^{i}{ }_{4}(\tau)=\hat{a}^{i}(\tau)
$$

where the functions $\hat{a}^{i}$ and $\hat{\omega}^{k}$ are obtained from the coefficients in the metric (10). Then take $e_{(\alpha)}^{\mu}(\tau)$ as the solution of the ordinary differential system

$$
\frac{\mathrm{d} e_{(\alpha)}^{\mu}}{\mathrm{d} \tau}=e_{(\beta)}^{\mu} \hat{\Omega}_{\alpha}^{\beta}(\tau)
$$

for some initial data $\left\{e_{(\alpha)}^{\mu}(0)\right\}_{\alpha=1 \ldots 4}$, that form an orthonormal tetrad, with $e_{(4)}^{\mu}(0)$ timelike. Due to the particular form of the matrix $\hat{\Omega}_{\alpha}^{\beta}$, it is obvious that $e_{(\alpha)}^{\mu}(\tau)$ is an orthonormal tetrad for all $\tau$ as well.

Being $e_{(4)}^{\mu}$ a timelike vector, consider a worldline $z^{\mu}(\tau)$ such that $\dot{z}^{\mu}(\tau)=e_{(4)}^{\mu}(\tau)$ and the matrix

$$
\Omega_{\nu}^{\mu}=\hat{\Omega}_{\alpha}^{\beta} e_{(\beta)}^{\mu} e_{\nu}^{(\alpha)}
$$

(indices are raised and lowered with $\eta_{\alpha \beta}=\operatorname{diag}[+1,+1,+1,-1]$ ). It is straightforward to see that the tetrad $e_{(\alpha)}^{\mu}(\tau)$ is GFW transported along $z^{\mu}(\tau)$ with an angular velocity $\Omega_{\nu}^{\mu}(\tau)$.

Consider finally the coordinate transformation

$$
\left(X^{i}, T\right) \longrightarrow x^{\mu}=z^{\mu}(T)+X^{j} e_{(j)}^{\mu}(T)
$$

whose Jacobian and inverse Jacobian are respectively given by

$$
\begin{gathered}
\mathrm{d} x^{\mu}=\left(\dot{z}^{\mu}(T)+e_{(\alpha)}^{\mu}(T) \hat{\Omega}_{j}^{\alpha}(T) X^{j}\right) \mathrm{d} T+e_{(j)}^{\mu}(T) \mathrm{d} X^{j} \\
\mathrm{~d} T=-\frac{e_{\mu(4)}(T) \mathrm{d} x^{\mu}}{1+\vec{X} \cdot \vec{a}(T)}, \quad \mathrm{d} X^{i}=\left(e_{\mu(i)}+\frac{e_{\mu(4)}(T) \hat{\Omega}_{j}^{i}(T) X^{j}}{1+\vec{X} \cdot \vec{a}(T)}\right) \mathrm{d} x^{\mu}
\end{gathered}
$$

Substituting the latter in the expression (10) we obtain that, in the coordinates $x^{\mu}$, the invariant interval is $\mathrm{d} s^{2}=\eta_{\mu \nu} \mathrm{d} x^{\mu} \mathrm{d} x^{\nu}$; hence $x^{\mu}$ are Lorentzian coordinates and $X^{i}, T$ are the GFW coordinates for the worldline $z^{\mu}(\tau)$ and the tetrad $e_{(\alpha)}^{\mu}$. 


\subsection{A geometric characterization of GFW coordinate systems}

We shall see now that, if the hypersurfaces $T=$ constant are hyperplanes in Minkowski spacetime and $X^{j}$ are Cartesian coordinates on these hyperplanes, then $\left(X^{j}, T\right)$ are GFW coordinates apart from a shift in the origin.

Proposition 2 Let $\left(X^{j}, T\right)$ be a system of coordinates such that the Minkowski metric spatial components are $g_{i j}=\delta_{i j}$, then: (a) the hypersurfaces $T=$ constant are flat, (b) the rank of their extrinsic curvature $K_{i j}$ is at most one, and (c) it satisfies that $\partial_{[i} K_{j] l}=0$.

Proof: The metric restricted to the hypersurfaces $T=$ constant, $\bar{g}_{i j}=\delta_{i j}$, is flat and the ambient metric is Minkowski metric. Then, if $K_{i j}$ is the second fundamental form, Gauss equation [30] implies that $K_{i[j} K_{l] k}=0$.

The latter equation having the same symmetries as a Riemann tensor and being three the number of effective dimensions, it is equivalent to its trace $(i l)$, that is:

$$
K_{i}^{j} K_{k}^{i}-K_{i}^{i} K_{k}^{j}=0
$$

which implies that $K_{i}^{j}$ has two eigenvalues, namely $K_{i}^{i}$ (simple) and 0 (double), hence $\operatorname{rank} K_{i}^{j} \leq 1$. The relation $\partial_{[i} K_{j] l}=0$ is a consequence of the Codazzi-Mainardi equation [30] and the fact that $X^{j}$ are Cartesian coordinates for the first fundamental form on the hypersurfaces $T=$ constant.

Proposition 3 If the metric spatial components are $g_{i j}=\delta_{i j}$ and the second fundamental form on $T=$ constant vanishes, then $\left(X^{j}, T\right)$ are $G F W$ coordinates apart from a shift in the origin.

Proof: Let us define $v_{i}=g_{4 i}$ and $N^{2}=\vec{v}^{2}-g_{44}$, then the inverse spacetime metric components are:

$$
g^{i j}=\delta_{i j}-N^{-2} v_{i} v_{j}, \quad g^{4 i}=N^{-2} v_{i}, \quad g^{44}=-N^{-2}=\operatorname{det}\left(g^{\mu \nu}\right) \neq 0
$$

and the connexion symbols are:

$$
\left.\begin{array}{lc}
\{i j \mid k\}=0, \quad\{i j \mid 4\}=\partial_{(i} v_{j)}, & \{4 i \mid j\}=\partial_{[i} v_{j]} \\
\{44 \mid i\}=\partial_{T} v_{i}+N \partial_{i} N+2 \vec{v} \cdot \partial_{i} \vec{v} \quad, & \{44 \mid 4\}=N \partial_{T} N+2 \vec{v} \cdot \partial_{T} \vec{v}
\end{array}\right\}
$$


The unit covector normal to the hypersurfaces $T=$ constant and the second fundamental form are respectively

$$
n_{a}=-N \delta_{a}^{4} \quad \text { and } \quad K_{i j}=-N \Gamma_{i j}^{4}=N^{-1} \partial_{(i} v_{j)}
$$

The vanishing of $K_{i j}$ then implies that $\partial_{i} v_{j}=W_{i j}$ is skewsymmetric, whose integrability conditions, $\partial_{l} W_{i j}=\partial_{i} W_{l j}$, combined with by the Jacobi identity, imply that $\partial_{j} W_{i l}=0$, that is

$$
W_{i j}=\epsilon_{i j k} \hat{\omega}^{k}(T) \quad \text { and therefore } \quad v_{i}=\epsilon_{i j k} X^{j} \hat{\omega}^{k}(T)+V_{i}(T)
$$

So far we have used the restrictions imposed by Gauss and Codazzi-Mainardi equations. We have still to exploit the vanishing of the components $R_{4 i 4 j}$. Including that $\partial_{(i} v_{j)}=0$ and the connexion symbols (21), we arrive at

$$
R_{4 i 4 j}=0 \quad \Leftrightarrow \quad \partial_{i j} N=0,
$$

that is

$$
\exists \vec{a}(T) \text { and } B(T) \text { such that } \quad N=B+\vec{X} \cdot \vec{a}
$$

and, provided that $B \neq 0$, the time coordinate $T$ can be redefined so that $B=1$.

Therefore, in these coordinates the Minkowski metric reads

$$
\mathrm{d} s^{2}=\mathrm{d} \vec{X}^{2}+2 \mathrm{~d} T \mathrm{~d} \vec{X} \cdot(\vec{X} \times \vec{\omega}+\vec{V})+\mathrm{d} T^{2}\left(-[1+\vec{X} \cdot \vec{a}]^{2}+[\vec{X} \times \vec{\omega}+\vec{V}]^{2}\right)
$$

Finally, if we shift the origin as: $\tilde{X}^{j}=X^{j}+M^{j}(T)$, where $\vec{M}(T)$ is a solution of $\partial_{T} \vec{M}+\vec{\omega} \times \vec{M}=\vec{V}$, the Minkowski spacetime interval in the new coordinates has the form $(10)$, i. e. $\left(\tilde{X}^{j}, T\right)$ are GFW coordinates.

\section{Generalized isometries}

Deriving a closed expression for the transformation relating two different GFW coordinate systems would imply to invert the transformation law (14), which is not feasible in general. However we can obtain expressions for infinitesimal transformations with the help of the notion of generalized isometry [29]. 
In a GFW coordinate system the invariant interval has a very specific shape (10) that involves six arbitrary functions $\hat{a}^{i}(T)$ and $\hat{\omega}^{j}(T)$. The general transformation formulae relating two GFW coordinate systems, $X^{\mu}=(\vec{X}, T) \longrightarrow X^{\prime \mu}=\left(\vec{X}^{\prime}, T^{\prime}\right)$, must preserve this overall shape but, perhaps, with a different sextuple of functions, $\left(\hat{a}^{\prime i}(t), \hat{\omega}^{\prime j}(t)\right)$. We shall call this transformation a generalized isometry [29] because the interval is:

$$
\mathrm{d} s^{2}=g_{\alpha \beta}\left(X^{\nu}, f_{I}(X)\right) \mathrm{d} X^{\alpha} \mathrm{d} X^{\beta}=g_{\alpha \beta}\left(X^{\prime \nu}, f_{I}^{\prime}\left(X^{\prime}\right)\right) \mathrm{d} X^{\prime \alpha} \mathrm{d} X^{\prime \beta}
$$

Although the functions $g_{\alpha \beta}\left(X, f_{I}\right)$ are the same, the metric coefficients, $\bar{g}_{\alpha \beta}(X)=g_{\alpha \beta}\left(X, f_{I}(X)\right)$, are different because the values $f_{I}(X)$ change to $f_{I}^{\prime}\left(X^{\prime}\right)$ in passing from one system to the other. This is the reason why the transformation is not actually an isometry and we need to introduce the notion of generalized isometry.

In the present case $f_{I}$ are six function that only depend on the coordinate $T$ and can be arranged as the skewsymmetric matrix

$$
\hat{\Omega}_{\alpha \beta}=\left(\begin{array}{c|c}
\epsilon_{i j k} \hat{\omega}^{k}(T) & \hat{a}^{i}(T) \\
\hline-\hat{a}^{j}(T) & 0
\end{array}\right)
$$

Consider now the infinitesimal transformation

$$
X^{\prime \alpha}=X^{\alpha}+\varepsilon \xi^{\alpha}(X), \quad f_{I}^{\prime}(X)=f_{I}(X)+\varepsilon \Phi_{I}(X)
$$

and therefore $f_{I}^{\prime}\left(X^{\prime}\right)=f_{I}(X)+\varepsilon\left[\Phi_{I}(X)+\xi^{\alpha} D_{\alpha} f_{I}(X)\right]$, where $X^{4}=T$ and $D_{\alpha}=\frac{\partial}{\partial X^{\alpha}}$. Substituting this in equation (24) and keeping only first order terms we obtain

$$
\xi^{\alpha} D_{\alpha} \bar{g}_{\mu \nu}+2 D_{(\nu} \xi^{\alpha} \bar{g}_{\mu) \alpha}+G_{\mu \nu}(X)=0
$$

where

$$
\bar{g}_{\mu \nu}(X)=g_{\mu \nu}\left(X, f_{I}(X)\right) \quad \text { and } \quad G_{\mu \nu}(X)=\sum_{I} \Phi_{I}\left(\frac{\partial g_{\mu \nu}}{\partial f_{I}}\right)_{\left(X, f_{I}(X)\right)}
$$

We have as many functions $\Phi_{I}$ as $f_{I}$ and they can be also arranged in the skewsymmetric matrix $\hat{F}_{\alpha \beta}$ as

$$
\hat{F}_{i j}=\epsilon_{i j k} \hat{\alpha}^{k}(T), \quad \hat{F}_{i 4}=-\hat{F}_{4 i}=\hat{b}^{i}(T)
$$


in much the same way as we did for the $f_{I}$ in the matrix (25).

Equation (27) can also be written as the generalized Killing equation

$$
\bar{\nabla}_{\mu} \xi_{\nu}+\bar{\nabla}_{\nu} \xi_{\mu}+G_{\mu \nu}=0
$$

where $\bar{\nabla}$ is the Levi-Civita connexion for $\bar{g}_{\mu \nu}$ and $\xi_{\mu}=\bar{g}_{\mu \nu} \xi^{\nu}$.

Notice that the infinitesimal transformation (26) acts on $X^{\alpha}$ and also on the functions $\hat{\Omega}_{\alpha \beta}$; hence the arena to represent their action is not Minkowski spacetime but rather the larger (infinite dimensional) manifold

$$
\mathcal{M}=\left\{\left(\vec{X}, T, \hat{\Omega}_{\alpha \beta}(t)\right) \in \mathbb{R}^{4} \times \mathcal{C}^{0}\left(\mathbb{R}, \mathbb{R}^{6}\right), \text { such that }|1+\vec{X} \cdot \vec{a}(T)|>|\vec{X} \times \vec{\omega}(T)|\right\}
$$

(the inequality is to ensure that the metric (10) is non-degenerate). The infinitesimal generator then looks like:

$$
\xi^{\alpha} D_{\alpha}+\int_{\mathbb{R}} \mathrm{d} t \hat{F}_{\alpha \beta}(t) \frac{\delta}{\delta \hat{\Omega}_{\alpha \beta}(t)}
$$

where $\xi^{\alpha}$ depends on $X^{j}, T$ and $\hat{\Omega}_{\mu \nu}(t)$.

The need of the additional information contained in the six functions $\hat{\Omega}_{\alpha \beta}(t)$ comes from the fact that the coordinates $(\vec{X}, T)$ are not enough to determine an event in spacetime unless we further indicate the family of GFW observers to which these coordinates belong. To fix $\hat{\Omega}_{\alpha \beta}(t)$ means choosing a subclass of GFW coordinate systems, that corresponding to GFW observers with the same intrinsic spacetime angular velocity. Each one is characterized by the initial values of the tetrad, $e_{(\alpha}^{\mu}(0)$, and the initial point in the origin worldline, $z^{\mu}(0)$, (the tetrad $e_{(\alpha}^{\mu}(\tau)$ and the worldline $z^{\mu}(\tau)$ are then obtained as in the proof of Proposition 1 in section 3). By the way, it is the same kind and number of parameters as for Poincaré group, although they are not Lorentzian observers because $\hat{\Omega}_{\alpha \beta}(t) \neq 0$.

The 4-dimensional submanifold $\mathcal{M}_{\hat{\Omega}}=\left\{\left(\vec{X}, T, \hat{\Omega}_{\alpha \beta}(t)\right) \in \mathcal{M}\right\}$ for a given $\hat{\Omega}_{\alpha \beta}(t)$ corresponds to the part of Minkowski spacetime which is accessible to the GFW observers of the class determined by this intrinsic spacetime angular velocity.

From a physical viewpoint it is worth to remark here that the magnitudes $\hat{\Omega}_{\alpha \beta}$ are measurable by the GFW observer by means of accelerometers $\left(\hat{a}_{j}=\hat{\Omega}_{4 j}\right)$ and gyrometers $\left(\hat{\Omega}_{j k}\right)$ along the observer's 
spatial axes. As their measures do not need resorting to anything external, these magnitudes are "absolute", hence there is no sense in referring to them as "relative acceleration" or "relative angular velocity" although one might be tempted to.

From the interval expression (10) we have that

$$
g_{i j}=\delta_{i j}, \quad g_{4 i}=\epsilon_{i j k} X^{j} \hat{\omega}^{k}, \quad g_{44}=(\vec{X} \times \vec{\omega})^{2}-(1+\vec{X} \cdot \vec{a})^{2}
$$

and, as it follows from (27),

$$
G_{i j}=0, \quad G_{4 i}=\epsilon_{i j k} X^{j} \hat{\alpha}^{k}, \quad \frac{1}{2} G_{44}=-(1+\vec{X} \cdot \vec{a}) \vec{X} \cdot \vec{b}+(\vec{X} \times \vec{\omega}) \cdot(\vec{X} \times \vec{\alpha})
$$

Using this and after a little algebra, the generalized Killing equation splits in several blocks as

$$
\begin{array}{ll}
\text { space: } & D_{(i} \xi_{j)}=0 \\
\text { cross: } & -(1+\vec{X} \cdot \vec{a})^{2} D_{i} \xi^{4}+D_{4} \xi_{i}+\epsilon_{l k}^{j} X^{l} \hat{\omega}^{k} D_{i} \xi_{j}-\xi_{j} \epsilon_{i k}^{j} \hat{\omega}^{k}+\epsilon_{i l k} X^{l} \hat{\alpha}^{k}=0 \\
\text { time: } & -(1+\vec{X} \cdot \vec{a}) D_{4}\left[(1+\vec{X} \cdot \vec{a}) \xi^{4}\right]+\epsilon_{l k}^{j} X^{l} \hat{\omega}^{k} D_{4} \xi_{j}+ \\
& \\
& \frac{1}{2} D_{j} g_{44}\left(\xi_{j}-\xi^{4} \epsilon_{j l k} X^{l} \hat{\omega}^{k}\right)+\frac{1}{2} G_{44}=0
\end{array}
$$

The general solution to the space block is

$$
\vec{\xi}=\vec{f}(T)+\vec{X} \times \vec{g}(T), \quad \text { where } \quad \vec{\xi}=\left(\xi_{1}, \xi_{2}, \xi_{3}\right),
$$

which, substituted in the cross equation yields

$$
-(1+\vec{X} \cdot \vec{a})^{2} D_{i} \xi^{4}+\dot{\phi}_{i}+\epsilon_{i l k} X^{l} \gamma^{k}=0,
$$

where a "dot" means derivative with respect to $T$, and

$$
\vec{\phi}=\dot{\vec{f}}+\vec{f} \times \omega, \quad \vec{\gamma}=\dot{\vec{g}}+\vec{g} \times \vec{\omega}+\vec{\alpha}
$$

Equation (37) giving all spatial derivatives of $\xi^{4}$, it carries some integrability conditions which after some algebra reduce to:

$$
\dot{\vec{g}}+\vec{g} \times \vec{\omega}+\vec{\alpha}-\vec{\phi} \times \vec{a}=0
$$


In such a case, the general solution of equation (37) is

$$
\xi^{4}=h(T)+\frac{\vec{X} \cdot \vec{\phi}}{1+\vec{a} \cdot \vec{X}}
$$

If we now substitute equations (36) and (39) in the time block (35), we obtain

$$
-(1+\vec{X} \cdot \vec{a})[\dot{h}+\vec{a} \cdot \vec{f}+\vec{X} \cdot(\dot{\vec{\phi}}+\dot{h} \vec{a}+h \dot{\vec{a}}+h \vec{a} \times \vec{\omega}+\vec{\phi} \times \vec{\omega}+\vec{g} \times \vec{a}+\vec{b})]=0
$$

where (31) and (32) have been included. Putting $\vec{\psi}=\vec{\phi}+h \vec{a}$, this amounts to

$$
\begin{aligned}
\dot{h}+\vec{a} \cdot \vec{f} & =0 \\
\dot{\vec{\psi}}+\vec{\psi} \times \omega+\vec{g} \times \vec{a}+\vec{b} & =0, \quad \text { with } \quad \vec{\psi}=\dot{\vec{f}}+\vec{f} \times \omega+h \vec{a}
\end{aligned}
$$

Together with (38), these equations constitute an ordinary differential system on the functions $h$, $\vec{f}$ and $\vec{g}$ that occur in the expressions (36) and (39) for $\xi_{i}$ and $\xi^{4}$. The solution is obtained in Appendix A in terms of 4-dimensional variables, namely the 4-vector $f^{\alpha}(T)=(\vec{f}, h)$ and the skewsymmetric tensor $M_{\alpha \beta}(T)$ formed with, respectively, $\vec{\psi}$ and $\vec{g}$ as the electric and magnetic parts. The solutions (67) and (68) depend on ten constant parameters, $f_{0}^{\mu}$ and $M_{\alpha \beta}^{0}$, plus six arbitrary one-variable functions, $\hat{F}_{\alpha \beta}(t)$.

Introducing then these solutions in the expressions (36) and (39), we have that the infinitesimal generator (30) can be written as

$$
\left[f^{\mu}(T)+M_{j}^{\mu}(T) X^{j}\right] \hat{D}_{\mu}+\int_{\mathbb{R}} \mathrm{d} t \hat{F}_{\alpha \beta}(t) \frac{\delta}{\delta \hat{\Omega}_{\alpha \beta}(t)}
$$

where

$$
\hat{D}_{i}=D_{i} \quad \text { and } \quad \hat{D}_{4}=\frac{1}{1+\vec{X} \cdot \vec{a}(T)}\left(D_{4}-\epsilon_{j k}^{i} X^{j} \hat{\omega}^{k}(T) D_{i}\right)
$$

i. e. $\hat{D}_{\mu}$ are a sort of orthonormalized partial derivatives.

\subsection{Infinitesimal generators and commutation relations}

Substituting now equation (69) in Appendix A into (43) and factoring out the ten constant parameters, $f_{0}^{\mu}$ and $M_{\alpha \beta}^{0}$, and the six arbitrary functions $\hat{F}_{\alpha \beta}(t)$, we can write the general infinitesimal generator as

$$
f_{0}^{\mu} \mathbf{P}_{\mu}+\frac{1}{2} M_{0}^{\alpha \beta} \mathbf{J}_{\alpha \beta}+\frac{1}{2} \int_{\mathbb{R}} \mathrm{d} t \hat{F}_{\alpha \beta}(t) \mathbf{A}_{(t)}^{\alpha \beta}
$$


where $M_{0}^{\alpha \beta}=M_{\mu \nu}^{0} \eta^{\alpha \mu} \eta^{\beta \nu}$ and

$$
\begin{aligned}
\mathbf{P}_{\mu} & =\Lambda_{\mu}^{\nu}(T) \hat{D}_{\nu}, \quad \mathbf{J}_{\alpha \beta}=-2 k_{[\alpha}(T, \vec{X}) \mathbf{P}_{\beta]} \\
\mathbf{A}_{(t)}^{\alpha \beta} & =\frac{\delta}{\delta \hat{\Omega}_{\alpha \beta}(t)}-2 \chi(t, T) \Lambda^{\mu[\alpha}(t) \Delta^{\beta]}(T, t, \vec{X}) \mathbf{P}_{\mu}
\end{aligned}
$$

with $\quad \chi(t, \tau)=\theta(t) \theta(\tau-t)-\theta(-t) \theta(t-\tau)$ and

$$
\begin{aligned}
k_{\beta}(T, \vec{X}) & =\Lambda_{\beta j}(T) X^{j}+\int_{0}^{T} \mathrm{~d} t^{\prime} \Lambda_{\beta 4}\left(t^{\prime}\right) \\
\Delta^{\nu}(T, t, \vec{X}) & =k_{\beta}(T, \vec{X}) \Lambda^{\beta \nu}(t)-\int_{0}^{t} \mathrm{~d} t^{\prime} G_{4}^{\nu}\left(t^{\prime}, t\right)
\end{aligned}
$$

as given by equations (70) and (71). (The matrices $\Lambda_{\mu}{ }^{\nu}(T)$ and $G_{\nu}{ }^{\mu}(T, t)$ are also defined in Appendix A.)

In order to better understand how these infinitesimal generators act on the manifold $\mathcal{M}$, we should think of it as sliced in the 4-dimensional submanifolds $\mathcal{M}_{\hat{\Omega}}$, each one characterized by a definite choice of the six functions $\hat{\Omega}_{\alpha \beta}(t)$. Any of these submanifolds is coordinated by $(\vec{X}, T)$ and endowed with the metric (10) and corresponds to the part of Minkowski spacetime that is accessible to the class of GFW observers defined by the given $\hat{\Omega}_{\alpha \beta}(t)$. The generators $\mathbf{P}_{\mu}$ and $\mathbf{J}_{\alpha \beta}$ act on -are tangent to - each slice and span the realization of Poincaré algebra for that particular class of GFW observers. On their turn, the generators $\mathbf{A}_{t}^{\alpha \beta}$ are transversal to the slicing and are connected with changes in the intrinsic proper acceleration and angular velocity of the observer.

Although the derivation of the Lie brackets between pairs of infinitesimal generators is tedious and intricated, it presents no conceptual subtlety and we shall not derive them explicitely here. An outline of their derivation is postponed to Appendix C. The commutation relations are:

$$
\left.\begin{array}{lll}
{\left[\mathbf{P}_{\mu}, \mathbf{P}_{\nu}\right]=0,} & {\left[\mathbf{J}_{\alpha \beta}, \mathbf{P}_{\mu}\right]=2 \eta_{\mu[\alpha} \mathbf{P}_{\beta]},} & {\left[\mathbf{J}_{\alpha \beta}, \mathbf{J}_{\mu \nu}\right]=2 \eta_{\mu[\alpha} \mathbf{J}_{\beta] \nu}-2 \eta_{\nu[\alpha} \mathbf{J}_{\beta] \mu}} \\
{\left[\mathbf{A}_{(t)}^{\alpha \beta}, \mathbf{P}_{\mu}\right]=0,} & {\left[\mathbf{A}_{(t)}^{\alpha \beta}, \mathbf{J}_{\alpha \beta}\right]=0,} & {\left[\mathbf{A}_{(t)}^{\alpha \beta}, \mathbf{A}_{\left(t^{\prime}\right)}^{\kappa \lambda}\right]=0}
\end{array}\right\}
$$

Thus, the algebra of the infinitesimal transformations connecting generalized Fermi-Walker coordinate systems is an abelian extension of Poincaré algebra. 


\subsection{A particular case: uniform proper acceleration without rotation}

We shall now restrain to the subclass of FW systems of coordinates such that $\hat{a}_{i}=$ constant and $\hat{\omega}_{i}=0$. The angular velocity matrix in body axes $\hat{\Omega}^{\mu}{ }_{\nu}$ then looks very simple and the invariant interval is

$$
\mathrm{d} s^{2}=-(1+\vec{a} \cdot \vec{X})^{2} \mathrm{~d} t^{2}+\mathrm{d} \vec{X}^{2}, \quad \vec{a}=\text { constant }
$$

To derive the infinitesimal transformations connecting two of these coordinate systems we must solve the generalized Killing equation (29) with all the $G_{\mu \nu}$ but one vanishing

$$
G_{44}=-2(1+\vec{a} \cdot \vec{X}) \vec{b} \cdot \vec{X}
$$

The infinitesimal generator (43) is in this case

$$
\left[f^{\mu}(T)+M_{j}^{\mu}(T) X^{j}\right] \hat{D}_{\mu}+\hat{a}_{i} \frac{\partial}{\partial \hat{a}_{i}}
$$

(The functional derivative in (43) has become a partial derivative because, on restricting to $\hat{a}_{i}=$ constant, the space on which the generator acts, namely

$$
\mathcal{M}_{A} \subset\left\{(\vec{X}, T, \vec{a}) \in \mathbb{R}^{7}, \text { such that } 1+\vec{X} \cdot \vec{a}(T)>0\right\}
$$

is finite dimensional.

The generalized Killing equations (33) to (35) reduce to

$$
D_{(i} \xi_{j)}=0, \quad-(1+\vec{X} \cdot \vec{a})^{2} D_{i} \xi^{4}+D_{4} \xi_{i}=0
$$

and

$$
-(1+\vec{X} \cdot \vec{a}) D_{4}\left[(1+\vec{X} \cdot \vec{a}) \xi^{4}\right]+\frac{1}{2} D_{j} g_{44} \xi_{j}+\frac{1}{2} G_{44}=0
$$

The solution is given in Appendix B and the infinitesimal generator depends on thirteen constant parameters

$$
f_{0}^{\mu} \mathbf{P}_{\mu}+\frac{1}{2} M_{0}^{\alpha \beta} \mathbf{J}_{\alpha \beta}+\hat{b}_{i} \mathbf{A}^{i}
$$


where

$$
\begin{aligned}
\mathbf{P}_{i} & =\left(\delta_{i}^{j}+\frac{\cosh a T-1}{a^{2}} \hat{a}_{i} \hat{a}^{j}\right) D_{j}-\frac{\sinh a T}{a(1+\vec{a} \cdot \vec{X})} \hat{a}_{i} D_{T} \\
\mathbf{P}_{4} & =\frac{\cosh a T}{(1+\vec{a} \cdot \vec{X})} D_{T}-\frac{\sinh a T}{a} \hat{a}^{j} D_{j} \\
\mathbf{J}_{\alpha \beta} & =-2 k_{[\alpha} \mathbf{P}_{\beta]}, \quad \text { with } \\
k_{i} & =X_{i}+\frac{\cosh a T-1}{a^{2}}(1+\vec{a} \cdot \vec{X}) \hat{a}_{i}, \quad k_{4}=-\frac{\sinh a T}{a}(1+\vec{a} \cdot \vec{X})
\end{aligned}
$$

and

$$
\begin{aligned}
\mathbf{A}^{i}= & \frac{\partial}{\partial \hat{a}_{i}}+\frac{\cosh a T-1}{a^{2}}\left[-(1+\vec{a} \cdot \vec{X}) \delta_{j}^{i}+X^{i} \hat{a}_{j}\right] D_{j}+ \\
& \left(\frac{\sinh a T-a T}{a^{3}} \hat{a}_{i}-X^{i} \frac{\sinh a T}{a(1+\vec{a} \cdot \vec{X})}\right) D_{T}
\end{aligned}
$$

where the results in Appendix B have been included. The commutation relations are similar to those given by (50) for the general case.

It is worth to consider now the limit $c \rightarrow \infty$, to compare with the extensions of Galilei algebra to include acceleration [8]. To obtain this limit we must first replace $a T$ and $\vec{a} \cdot \vec{X}$ with $a T / c$ and $\vec{a} \cdot \vec{X} / c^{2}$ and also add the necessary $c^{n}$ factors in formulae (51) to (56) to make them dimensionally consistent.

Defining then $\mathbf{H}=c \mathbf{P}_{4}$ and $\mathbf{K}_{i}=c \mathbf{J}_{4 i}$ and taking the limit $c \rightarrow \infty$, we obtain

$$
\begin{aligned}
& \mathbf{P}_{i}=D_{i}, \quad \mathbf{H}=D_{T}-T \hat{a}^{j} D_{j}, \quad \mathbf{K}_{i}=T \mathbf{P}_{i}, \\
& \mathbf{J}_{i}=\varepsilon_{i j l}\left(X^{j}+\frac{T^{2}}{2} \hat{a}^{j}\right) D_{l}, \quad \mathbf{A}^{i}=\frac{\partial}{\partial \hat{a}_{i}}-\frac{T^{2}}{2} \mathbf{P}_{i}
\end{aligned}
$$

and the only non-vanishing commutation relations are

$$
\left[\mathbf{H}, \mathbf{K}_{i}\right]=\mathbf{P}_{i}, \quad\left[\mathbf{J}_{i}, \mathbf{P}_{j}\right]=-\varepsilon_{i j l} \mathbf{P}_{l}, \quad\left[\mathbf{J}_{i}, \mathbf{J}_{j}\right]=-\varepsilon_{i j l} \mathbf{J}_{l}, \quad\left[\mathbf{J}_{i}, \mathbf{K}_{j}\right]=-\varepsilon_{i j l} \mathbf{K}_{l},
$$

as it corresponds to an Abelian extension of Galilei algebra and consistently with (50). 
Notice that these differ from the commutation relations obtained by Lukierski et al. [8], where the generators of the exended algebra are:

$$
\mathbf{P}_{i}^{*}=D_{i}, \quad \mathbf{H}^{*}=D_{T}, \quad \mathbf{J}_{i}^{*}=\varepsilon_{i j l} X^{j} D_{l}, \quad \mathbf{K}_{i}^{*}=T D_{i}, \quad \mathbf{A}_{i}^{*}=T^{2} D_{i}
$$

and one of the commutation relations is different, namely $\left[\mathbf{J}_{i}^{*}, \mathbf{A}_{j}^{*}\right]=-\varepsilon_{i j l} \mathbf{A}_{l}^{*}$.

One possible reason for this disagreement is that the setting of $\mathrm{FW}$ coordinates assigns a crucial role to simultaneity with respect to the inertial reference system instantaneously comoving with the origin; whence the term $-T \hat{a}^{j} D_{j}$ in the generator of time translations, whereas in Lukierski's work simultaneity is absolute. Furthermore, in the present approach the realization of Poincaré group acts on the space $\mathcal{M}_{A}$, coordinated by $\left(X^{j}, T, \hat{a}^{l}\right)$, whence the occurrence of $\partial / \partial \hat{a}_{i}$ in the acceleration boosts (58). The proper acceleration $\hat{a}^{l}$ of the origin of coordinates is absolute, as discussed in section 4 , and if the acceleration does not vanish, the simultaneity hyperplanes are not parallel to each other and a term $-T \hat{a}^{j} D_{j}$ occurs in the time translation generator.

\section{Conclusion}

We have introduced a class of reference frames with arbitrary translational and rotational motion, namely generalized Fermi-Walker frames. Each one is determined by the worldline of its spatial origin and a triad of spatial comoving axes with an arbitrary rotational motion.

Each GFW system of coordinates, $\left(T, X^{1}, X^{2}, X^{3}\right)$ is characterized by six functions of proper time, $\hat{a}_{i}(\tau)$ and $\hat{\omega}_{l}(\tau)$, respectively the components of the [proper] acceleration of the origin and the angular velocity of the spatial triad with respect to the comoving axes. These quantities, which are better handled as the skewsymmetric matrix $\hat{\Omega}_{\alpha \beta}(\tau)$ - see equation (25) - are measurable from inside the frame, i. e. without referring to anything external, by means of accelerometers and gyrometers.

The transformations connecting the coordinates of any pair of frames in the GFW class preserve the form (7) of the spacetime interval, maybe with different functions $\hat{\Omega}_{\alpha \beta}(\tau)$. Thus we refer to these transformations as generalized isometries. Infinitesimal generalized isometries satisfy the generalized Killing equation (37), whose solution is an infinite dimensional Lie algebra that contains 
Poincaré algebra and acceleration boosts plus rotational motions as well. A close look at the commutation relations reveals that it is an Abelian extension of Poincaré algebra. From the mathematical standpoint, the resulting structure, namely the direct sum of Poincaré algebra and an infinite dimensional abelian algebra, is rather trivial and dull. However it is somewhat surprising because, just as velocity boosts $\mathbf{J}_{4 j}$ do not commute with each other nor with rotations $\mathbf{J}_{i j}$ or translations $\mathbf{P}_{\mu}$, one would expect something similar for acceleration boosts and angular velocity generators, i. e. $\mathbf{A}_{t}^{\alpha \beta}$, but the effective calculation leads to the "counterintuitive" relations (50).

Perhaps this unexpected commutativity is caused by the fact that the parametrization $\hat{\Omega}_{\alpha \beta}(t)$ we have chosen for the extension of Poincaré algebra is somewhat intrinsic. Indeed, $\hat{\Omega}_{4 i}(t)$ and $\hat{\Omega}_{j k}(t)$ are, respectively, the components of proper acceleration and angular velocity in the triad of body axes, which are carried by the GFW observer, without reference to anything external.

We have particularly considered the case of reference systems with uniform proper acceleration without rotation because they have some historical interest, they are indeed the accelerated reference systems introduced by Einstein in his pioneer paper [1], as it has been proved elsewhere [17]. Moreover, we have taken the non-relativistic limit, $c \rightarrow \infty$, and have compared it with the extension of Galilei algebra obtained by Lukierski et al.[8] in a Newtonian framework. As a result, both extensions are different because both the generators and their commutation relations are. In our view, this is due to a condition which is crucial in our approach: at any moment there exists an inertial system which is instantaneously at rest with an accelerated non-rotating systems of ours. The influence of this, relativistic in origin, condition persists in the generators $\mathbf{H}, \mathbf{J}_{i}$ and $\mathbf{A}^{j}$ even after taking the limit $c \rightarrow \infty$.

It is also remarkable that the notion of generalized isometry [29] permits to go beyond Kretschmann's idea that, since special relativity admits the widest isometry group, it contains the largest relativity postulate. Our approach here has led to an intermediate group, namely the group of generalized isometries of the interval (7), which is larger than Poincaré group but much smaller than the whole diffeomorfism group. On the other hand, this intermediate group can be seen as the special relativistic counterpart of the Galilei group extensions considered elsewhere [7], [8],[9]. 


\section{Acknowledgement}

Funding for this work was partially provided by the Spanish MINECO under MDM-2014-0369 of ICCUB (Unidad de Excelencia 'María de Maeztu') and by Ministerio de Economia y Competitividad and ERDF (project ref. FPA2016-77689-C2-2-R).

\section{Appendix A: Solving the generalized Killing equation}

We here solve equations (38), (41) and (42), that we first arrange as

$$
\left.\left.\begin{array}{l}
\dot{\vec{f}}+h \vec{a}+\vec{f} \times \vec{\omega}=\vec{\psi} \\
\dot{h}+\vec{f} \cdot \vec{a}=0
\end{array}\right\} \begin{array}{l}
\dot{\vec{\psi}}+\vec{\psi} \times \vec{\omega}+\vec{g} \times \vec{a}=-\vec{A} \\
\dot{\vec{g}}-\vec{\psi} \times \vec{a}+\vec{g} \times \vec{\omega}=-\vec{\alpha}
\end{array}\right\}
$$

If we put $f^{\alpha}=(\vec{f}, h)$ and $\psi^{\alpha}=(\vec{\psi}, 0)$, the first pair of equations can be written as

$$
\dot{f}^{\alpha}+\hat{\Omega}_{\beta}^{\alpha} f^{\beta}=\psi^{\alpha}
$$

where $\hat{\Omega}_{\beta}^{\alpha}$ is the matrix (17).

Consider now the Lorentz matrix $\Lambda_{\alpha}^{\mu}(T)=e_{(\alpha)}^{\mu}(T)$ which is a solution of equation (18) with the unit matrix as initial data

$$
\dot{\Lambda}_{\alpha}^{\mu}=\Lambda_{\beta}^{\mu} \hat{\Omega}_{\alpha}^{\beta}, \quad \Lambda_{\alpha}^{\mu}(0)=\delta_{\alpha}^{\mu}
$$

Including that $\hat{\Omega}_{\nu}{ }^{\beta}=-\hat{\Omega}^{\beta}{ }_{\nu}$, we have that the inverse matrix, $\Lambda_{\mu}{ }^{\alpha}=\eta_{\mu \nu} \Lambda^{\nu}{ }_{\beta} \eta^{\beta \alpha}$, is a solution of

$$
\dot{\Lambda}_{\mu}^{\alpha}+\hat{\Omega}_{\beta}^{\alpha} \Lambda_{\mu}^{\beta}=0
$$

Hence, $C^{\nu} \Lambda_{\nu}{ }^{\alpha}(T)$, with $C^{\nu}$ constant, is a solution of the homogeneous part of equation (60). The complete equation can be solved by the method of variation of constants and we so obtain

$$
f^{\alpha}(T)=f_{0}^{\nu} \Lambda_{\nu}^{\alpha}(T)+\int_{0}^{T} \mathrm{~d} t G_{\nu}^{\alpha}(T, t) \psi^{\nu}(t)
$$

where

$$
G_{\rho}^{\mu}(\tau, t)=\Lambda_{\nu}^{\mu}(\tau) \Lambda_{\rho}^{\nu}(t)=G_{\rho}{ }^{\mu}(t, \tau)
$$


acts as a kind of matrix Green function and

$$
\partial_{T} G^{\alpha}{ }_{\nu}(T, t)=\hat{\Omega}_{\lambda}^{\alpha}(T) G^{\lambda}{ }_{\nu}(T, t)
$$

This solves the first pair of equations (59) provided that $\vec{\psi}(T)$ is known.

It is worth noticing that, except in the case of one-directional motion, the matrices $\Lambda_{\nu}{ }^{\mu}(T), T \in \mathbb{R}$ are not in general a one-parameter subgroup of Lorentz group; however the matrices $G^{\alpha}{ }_{\nu}(T, t)$ do have the group property:

$$
G_{\nu}^{\alpha}(T, t) G_{\lambda}^{\nu}\left(t, t^{\prime}\right)=G_{\lambda}^{\alpha}\left(T, t^{\prime}\right)
$$

and also

$$
G^{\alpha}{ }_{\nu}(T, t)=G_{\nu}^{\alpha}(t, T) \quad \text { and } \quad G^{\alpha}{ }_{\nu}(t, t)=\delta_{\nu}^{\alpha}
$$

To solve the second pair of equations we first organize the unknowns $\vec{g}$ and $\vec{\psi}$ as a skewsymmetric matrix $M_{\alpha \beta}$, with

$$
M_{i j}=\epsilon_{i j k} g^{k}, \quad M_{i 4}=-M_{4 i}=\psi^{i}
$$

and also

$$
F_{i j}=\epsilon_{i j k} \hat{\omega}^{k}, \quad M_{i 4}=-M_{4 i}=A^{i}
$$

so that equations (59) become

$$
\dot{M}_{\alpha \beta}=M_{\rho \beta} \hat{\Omega}_{\alpha}^{\rho}+M_{\alpha \rho} \hat{\Omega}_{\beta}^{\rho}-\hat{F}_{\alpha \beta}
$$

whose general solution is

$$
M_{\alpha \beta}(T)=M_{\mu \nu}^{0} \Lambda_{\alpha}^{\mu}(T) \Lambda_{\beta}^{\nu}(T)-\int_{0}^{T} \mathrm{~d} t \hat{F}_{\mu \nu}(t) G_{\alpha}{ }^{\mu}(T, t) G_{\beta}{ }^{\nu}(T, t)
$$

where $G_{\alpha}{ }^{\mu}$ is obtained by raising/lowering the indices in the matrix Green function (63) and $M_{\mu \nu}^{0}=$ $M_{\nu \mu}^{0}$ are constant.

Finally, as $\psi^{\nu}(t)=M^{\nu}{ }_{4}(t)$, equation (62) leads to

$$
f^{\alpha}(T)=f_{0}^{\nu} \Lambda_{\nu}^{\alpha}(T)+M_{\mu \lambda}^{0} \Lambda^{\mu \alpha}(T) \int_{0}^{T} \mathrm{~d} t \Lambda_{4}^{\lambda}(t)-\int_{0}^{T} \mathrm{~d} t \hat{F}_{\mu \lambda}(t) G^{\alpha \mu}(T, t) \int_{t}^{T} \mathrm{~d} t^{\prime} G_{4}{ }^{\lambda}\left(t^{\prime}, t\right)
$$

where (65) has been included. 
These expressions, (67) and (68), are to be substituted into the generator (43) and we obtain

$$
f^{\mu}(T)+M^{\mu}{ }_{j} X^{j}=f_{0}^{\nu} \Lambda_{\nu}{ }^{\mu}(T)+M_{\alpha \beta}^{0} \Lambda^{\alpha \mu}(T) k^{\beta}(T, \vec{X})-\int_{0}^{T} \mathrm{~d} t \hat{F}_{\alpha \beta}(t) G^{\mu \alpha}(T, t) \Delta^{\beta}(T, t, \vec{X})
$$

where the auxiliary variables

$$
\begin{aligned}
k^{\beta}(T, \vec{X}) & =\Lambda_{j}^{\beta}(T) X^{j}+\int_{0}^{T} \mathrm{~d} t \Lambda_{4}^{\beta}(t) \\
\Delta^{\beta}(T, t, \vec{X}) & =\Lambda_{\nu}{ }^{\beta}(t)\left[k^{\nu}(T, \vec{X})-\int_{0}^{t} \mathrm{~d} t^{\prime} \Lambda^{\nu}{ }_{4}\left(t^{\prime}\right)\right]
\end{aligned}
$$

have been introduced.

\section{Appendix B: Non-rotating uniformly accelerated systems}

We here particularize the results in Appendix A to the subclass of FW coordinates with uniform acceleration (without rotation). In the body axes the angular 4 -velocity matrix $\hat{\Omega}_{\alpha}^{\mu}$ is

$$
\hat{\Omega}_{4}^{i}=\hat{\Omega}_{i}^{4}=\hat{a}_{i}, \quad \hat{\Omega}_{j}^{i}=0, \quad i, j=1 \ldots 3
$$

It can be easily checked that the general solution to equation (61) is

$$
\Lambda_{\nu}^{\mu}(t)=\frac{\cosh (a t)-1}{a^{2}} H_{\nu}^{\mu}+\frac{\sinh (a t)}{a} \hat{\Omega}_{\nu}^{\mu}+\delta_{\nu}^{\mu}
$$

where $a^{2}=\vec{a} \cdot \vec{a}$ and $H_{\nu}^{\mu}=\hat{\Omega}_{\alpha}^{\mu} \hat{\Omega}_{\nu}^{\alpha}$.

In this particular case the matrices $\Lambda_{\nu}^{\mu}(t)$ are a 1-parameter subgroup of Lorentz group and the Green functions (64) are:

$$
G_{\nu}^{\mu}(\tau, t)=\Lambda_{\nu}^{\mu}(t-\tau)=\Lambda_{\nu}^{\mu}(\tau-t)
$$

Including this, equations (70), (71) and (69) become

$$
\begin{aligned}
k^{\beta}(T, \vec{X}) & =\Lambda^{\beta}{ }_{j}(T) X^{j}+\frac{\sinh (a T)}{a} \delta_{4}^{\beta}+\frac{\cosh (a T)-1}{a^{2}} \hat{\Omega}^{\beta}{ }_{4} \\
\Delta^{\beta}(T, t, \vec{X}) & =k^{\beta}(T-t, \vec{X})
\end{aligned}
$$


and

$$
f^{\mu}(T)+M_{j}^{\mu} X^{j}=f_{0}^{\nu} \Lambda_{\nu}^{\mu}(T)+M_{\alpha \beta}^{0} \Lambda^{\alpha \mu}(T) k^{\beta}(T, \vec{X})-2 A_{i} \int_{0}^{T} \mathrm{~d} t \Lambda^{[i \mu}(t) k^{4]}(t, \vec{X})
$$

or, including (72) and (73) and after some algebra,

$$
\begin{aligned}
& f^{\mu}(T)+M_{j}^{\mu} X^{j}=f_{0}^{\nu} \Lambda_{\nu}{ }^{\mu}(T)+M_{\alpha \beta}^{0} \Lambda^{\alpha \mu}(T) k^{\beta}(T, \vec{X})+\hat{a}^{\mu}(\vec{b} \cdot \vec{X}) \frac{\cosh a T-1}{a^{2}} \\
& \quad+\delta_{4}^{\mu}\left[\frac{\sinh a T-a T}{a^{3}}(\vec{b} \cdot \vec{a})(1+\vec{a} \cdot \vec{X})-\frac{\sinh a T}{a}(\vec{b} \cdot \vec{X})\right]-A^{\mu} \frac{\cosh a T-1}{a^{2}}(1+\vec{a} \cdot \vec{X})
\end{aligned}
$$

\section{Appendix C: Commutation relations}

Here we outline the main traits in the derivation of the commutation relations (50).

From (44) we have that the only non-vanishing Lie brackets among $\hat{D}_{\mu}$ 's are

$$
\left[\hat{D}_{i}, \hat{D}_{4}\right]=\frac{1}{1+\vec{X} \cdot \vec{a}(T)} \hat{\Omega}_{i}^{\sigma}(T) \hat{D}_{\sigma}
$$

wich, combined with

$$
\hat{D}_{4} \Lambda_{\nu}^{\beta}(T)=\frac{1}{1+\vec{X} \cdot \vec{a}(T)} \Lambda_{\nu}^{\rho}(T) \hat{\Omega}_{\rho}^{\beta}(T)
$$

readily leads to

$$
\left[\mathbf{P}_{\mu}, \mathbf{P}_{\nu}\right]=0
$$

Now, from (48) we easily obtain that

$$
\mathbf{P}_{\mu} k_{\beta}=\eta_{\mu \beta}
$$

which, combined with the second equation (46) and (77), immediately yields

$$
\left[\mathbf{J}_{\alpha \beta}, \mathbf{P}_{\mu}\right]=2 \eta_{\mu[\alpha} \mathbf{P}_{\beta]}, \quad\left[\mathbf{J}_{\alpha \beta}, \mathbf{J}_{\mu \nu}\right]=2 \eta_{\mu[\alpha} \mathbf{J}_{\beta] \nu}-2 \eta_{\nu[\alpha} \mathbf{J}_{\beta] \mu}
$$

That is, the infinitesimal generators $\mathbf{P}_{\mu}$ and $\mathbf{J}_{\alpha \beta}$ span Poincaré algebra.

To derive the commutators of these with the generators $\mathbf{A}_{t}^{\alpha \beta}$, we shall use that

$$
D_{4} \chi(t, T)=\delta(T-t) \quad \text { and } \quad \mathbf{P}_{\mu} \Delta^{\beta}(T, t, \vec{X})=\Lambda_{\mu}{ }^{\beta}(t)
$$


Moreover, form (61) we have that

$$
D_{4}\left(\frac{\delta \Lambda_{\mu}{ }^{\nu}(T)}{\delta \hat{\Omega}_{\alpha \beta}(t)}\right)=\frac{\delta \Lambda_{\mu}{ }^{\rho}(T)}{\delta \hat{\Omega}_{\alpha \beta}(t)} \hat{\Omega}_{\rho}^{\nu}(T)+2 \delta(T-t) \Lambda_{\mu}{ }^{[\alpha}(t) \eta^{\beta] \nu}
$$

which can be integrated to obtain:

$$
\frac{\delta \Lambda_{\mu}{ }^{\nu}(T)}{\delta \hat{\Omega}_{\alpha \beta}(t)}=2 \chi(t, T) \Lambda_{\mu}{ }^{[\alpha}(t) G^{\beta] \nu}(t, T)
$$

We also need that

$$
\left[\hat{D}_{4}, \frac{\delta}{\delta \hat{\Omega}_{\alpha \beta}(t)}\right]=\frac{2}{1+\vec{X} \cdot \vec{a}(T)} \delta(T-t) X^{j} \delta_{j}^{[\beta} \eta^{\alpha] \rho} \hat{D}_{\rho}
$$

Then, combining equations (79) to (81) we easily arrive at

$$
\left[\mathbf{P}_{\mu}, \mathbf{A}_{t}^{\alpha \beta}\right]=0
$$

and, including (46) and the fact that

$$
\frac{\delta k_{\mu}(T, \vec{X})}{\delta \hat{\Omega}_{\alpha \beta}(t)}=2 \chi(t, T) \Lambda_{\mu}^{[\alpha}(t) \Delta^{\beta]}(T, t, \vec{X}),
$$

we readily obtain that $\mathbf{A}_{t}^{\alpha \beta} k_{\mu}=0$ which, combined with (46) and (82) yields

$$
\left[\mathbf{J}_{\mu \nu}, \mathbf{A}_{t}^{\alpha \beta}\right]=0
$$

Finally, to calculate the commutators between pairs of generators of the kind $\mathbf{A}_{t}^{\alpha \beta}$, we realise that, from (47) and (83),

$$
\mathbf{A}_{t}^{\mu \nu}=\frac{\delta}{\delta \hat{\Omega}_{\mu \nu}(t)}-\frac{\delta k^{\rho}(T, \vec{X})}{\delta \hat{\Omega}_{\alpha \beta}(t)} \mathbf{P}_{\rho}
$$

and therefore

$$
\begin{aligned}
{\left[\mathbf{A}_{t}^{\mu \nu}, \mathbf{A}_{t^{\prime}}^{\alpha \beta}\right]=} & \left(-\frac{\delta^{2} k^{\sigma}(T, \vec{X})}{\delta \hat{\Omega}_{\mu \nu}(t) \delta \hat{\Omega}_{\alpha \beta}\left(t^{\prime}\right)}+\frac{\delta^{2} k^{\sigma}(T, \vec{X})}{\delta \hat{\Omega}_{\alpha \beta}\left(t^{\prime}\right) \delta \hat{\Omega}_{\mu \nu}(t)}\right) \mathbf{P}_{\sigma} \\
& -\frac{\delta k^{\sigma}(T, \vec{X})}{\delta \hat{\Omega}_{\alpha \beta}\left(t^{\prime}\right)}\left[\frac{\delta}{\delta \hat{\Omega}_{\mu \nu}(t)}, \mathbf{P}_{\sigma}\right]+\frac{\delta k^{\sigma}(T, \vec{X})}{\delta \hat{\Omega}_{\mu \nu}(t)}\left[\frac{\delta}{\delta \hat{\Omega}_{\alpha \beta}\left(t^{\prime}\right)}, \mathbf{P}_{\sigma}\right] \\
& +\left[\frac{\delta k^{\sigma}(T, \vec{X})}{\delta \hat{\Omega}_{\mu \nu}(t)} \mathbf{P}_{\sigma}, \frac{\delta k^{\rho}(T, \vec{X})}{\delta \hat{\Omega}_{\alpha \beta}\left(t^{\prime}\right)} \mathbf{P}_{\rho}\right]
\end{aligned}
$$


Now, as cross partial derivatives are equal, the first term in the right hand side vanishes. Furthermore, as $\mathbf{P}_{\sigma}$ and $\mathbf{A}_{t^{\prime}}^{\alpha \beta}$ commute, we have that

$$
\left[\frac{\delta}{\delta \hat{\Omega}_{\alpha \beta}\left(t^{\prime}\right)}, \mathbf{P}_{\sigma}\right]=-\mathbf{P}_{\sigma}\left(\frac{\delta k^{\rho}(T, \vec{X})}{\delta \hat{\Omega}_{\alpha \beta}\left(t^{\prime}\right)}\right) \mathbf{P}_{\rho}
$$

which, substituted in (86) yields

$$
\left[\mathbf{A}_{t}^{\mu \nu}, \mathbf{A}_{t^{\prime}}^{\alpha \beta}\right]=0
$$

\section{Appendix D: The group of transformations}

As the domain of any particular GFW system is not in general the whole Minkowski spacetime, the group of transformations connecting these systems is rather of local nature and some attention must be paid to the domains of such transformation maps.

A given GFW system $\mathcal{K}$ is characterized by $\left(z^{\mu}(\tau), e_{(i)}^{\nu}(\tau)\right)$, i. e. the origin worldline and the triad of spatial axes; it has also associated an angular 4-velocity $\hat{\Omega}_{\alpha \beta}$ in body axes [see eq. (17)]. To obtain the $\mathcal{K}$-coordinates $X^{\nu}=\left(X^{j}, T\right)$ of an event $x^{\mu}$ the relation $(8)$

$$
\left(T, X^{l}\right) \longrightarrow x^{\mu}=z^{\mu}(T)+X^{l} e_{(l)}^{\mu}(T),
$$

must be inverted. Generally an explicit inversion is not possible but, according to the inverse function theorem, it can be done whenever the Jacobian does not vanish. The latter can be easily derived from the metric (10) and it is

$$
\operatorname{det}\left(\frac{\partial x^{\nu}}{\partial X^{\mu}}\right)=1+\vec{X} \cdot \vec{a}(T)
$$

For those values $X^{l}$ such that the worldline $z^{\mu}(T)+X^{l} e_{(l)}^{\mu}(T)$ is timelike, that is

$$
[1+\vec{X} \cdot \vec{a}(T)]^{2}>(\vec{X} \times \vec{\omega})^{2}
$$

where the metric (10) has been included, the Jacobian does not vanish and (17) can be inverted. Consider then the open set

$$
\mathcal{V}_{\mathcal{K}}=\left\{\left(X^{j}, T\right) \in \mathbb{R}^{4}|1+\vec{X} \cdot \vec{a}(T)>| \vec{X} \times \vec{\omega} \mid\right\}
$$


and the map

$$
\left.\begin{array}{rl}
\varphi_{\mathcal{K}}: V_{\mathcal{K}} & \longrightarrow \mathcal{D}_{\mathcal{K}} \\
X^{\nu} & \longrightarrow z^{\mu}(T)+X^{l} e_{(l)}^{\mu}(T)
\end{array}\right\}
$$

where the range $\mathcal{D}_{\mathcal{K}}$ has been chosen so that $\varphi_{\mathcal{K}}$ is exhaustive and it corresponds to the "wedge" in Minkowski spacetime that is accessible to the GFW system $\mathcal{K}$. The "sizes" of $\mathcal{V}_{\mathcal{K}}$ and $\mathcal{D}_{\mathcal{K}}$ depend on $\hat{\Omega}_{\alpha \beta}$ and not on the origin or the initial orientation of the GFW transported tetrad.

As the Jacobian of this map is positive in the whole domain $\mathcal{V}_{\mathcal{K}}$, it has an inverse $\psi_{\mathcal{K}}: \mathcal{D}_{\mathcal{K}} \longrightarrow \mathcal{V}_{\mathcal{K}}$ and $X^{\nu}=\psi_{\mathcal{K}}\left(x^{\mu}\right)$ are the $\mathcal{K}$-coordinates of the event $x^{\mu}$ and

Let $\mathcal{K}$ and $\mathcal{K}^{\prime}$ two GFW systems. The $\mathcal{K}^{\prime}$-coordinates of an event $x^{\mu} \in \mathcal{D}_{\mathcal{K}^{\prime}}$ are

$$
X^{\prime \nu}=\psi_{\mathcal{K}^{\prime}}\left(x^{\mu}\right) \in \mathcal{V}_{\mathcal{K}^{\prime}}
$$

and, if $x^{\mu} \in \mathcal{D}_{\mathcal{K}}$, then $x^{\mu}=\varphi_{\mathcal{K}}\left(X^{\nu}\right)$, where $X^{\nu} \in \mathcal{V}_{\mathcal{K}}$ are the $\mathcal{K}$-coordinates of $x^{\mu}$.

Thus, if $x^{\mu} \in \mathcal{D}_{\mathcal{K}} \cap \mathcal{D}_{\mathcal{K}^{\prime}}$, then $X^{\prime \nu}=\psi_{\mathcal{K}^{\prime}}\left(\varphi_{\mathcal{K}}\left(X^{\mu}\right)\right)=\Phi_{\mathcal{K} \mathcal{K}^{\prime}}\left(X^{\mu}\right)$, where we have written

$$
\Phi_{\mathcal{K} \mathcal{K}^{\prime}}=\psi_{\mathcal{K}^{\prime}} \circ \varphi_{\mathcal{K}}
$$

The domain of this product map is $X^{\mu} \in \psi_{\mathcal{K}}\left(\mathcal{D}_{\mathcal{K}} \cap \mathcal{D}_{\mathcal{K}^{\prime}}\right)=\mathcal{V}_{\mathcal{K}} \cap \psi_{\mathcal{K}}\left(\mathcal{D}_{\mathcal{K}^{\prime}}\right)=\mathcal{V}_{\mathcal{K}^{\prime}}$ whereas its range is $X^{\prime \nu} \in \psi_{\mathcal{K}^{\prime}}\left(\mathcal{D}_{\mathcal{K}} \cap \mathcal{D}_{\mathcal{K}^{\prime}}\right)=\psi_{\mathcal{K}^{\prime}}\left(\mathcal{D}_{\mathcal{K}}\right) \cap \mathcal{V}_{\mathcal{K}^{\prime}}=\mathcal{V}_{\mathcal{K}^{\prime} \mathcal{K}}$.

It is obvious that $\mathcal{V}_{\mathcal{K} \mathcal{K}^{\prime}}$ is an open subset of $\mathcal{V}_{\mathcal{K}}$.

The transformation connecting $\mathcal{K}$-coordinates and $\mathcal{K}^{\prime}$-coordinates is the map

$$
\Phi_{\mathcal{K} \mathcal{K}^{\prime}}: \mathcal{V}_{\mathcal{K} \mathcal{K}^{\prime}} \longrightarrow \mathcal{V}_{\mathcal{K}^{\prime} \mathcal{K}}
$$

which by construction is bijective.

It follows straightforward that the class of all these maps is a group which extends Poincaré group. Indeed, as commented in section 2, inertial systems are a particular instance of GFW systems , when $\hat{\Omega}_{\alpha \beta}=0$ and therefore the origin worldline is straight and the tetrad is constant. If $\mathcal{K}$ is such a system, as $\hat{a}_{i}=\hat{\omega}_{j}=0$, there is no restriction either on the coordinates domain or wedge and $\mathcal{V}_{\mathcal{K}}=\mathcal{D}_{\mathcal{K}}=\mathbb{R}^{4}$. If $\mathcal{K}$ and $\mathcal{K}^{\prime}$ are two inertial systems it is thus obvious that $\Phi_{\mathcal{K} \mathcal{K}^{\prime}}$ is the Poincaré transformation connecting both systems. 
ExtendedPrinciple6 v0.tex

\section{References}

[1] Einstein A, Jahrb Rad Elektr 4 (1907) 411

[2] Kretschmann E, Ann Phys Lpz 53 (1917) 575

[3] Fock V A, The Theory of Space, Time and Gravitation, McMillan (1964)

[4] Antoci S and Liebscher D E, "The group aspect in the physical interpretation of general relativity theory", arXiv:gr-qc/0910.2073

[5] Ellis G F R and Matravers D R, Gen Relativ Gravit 27 (1995) 777

[6] Zalaletdinov R, Tavakol R and Ellis G F R, Gen Relativ Gravit 28 (1996) 1251

[7] Duval C, Class Quantum Grav 10 (1993) 2217 (arXiv:0903.1641 [math-ph])

[8] Lukierski J, Stichel P C and Zakrzewski W J, Phys Lett B650 (2007) 203 (arXiv:hepth/0702179)

[9] Andringa1 R, Bergshoeff E, Gomis J and de Roo M, Class Quantum Grav 29 (2012) 235020

[10] B Mashhoon, Annalen der Physik 18 (2009) 640; C Chicone and B Mashoon, Phys Rev D74 (2006) 064019

[11] Mashhoon B, Int Journal Math Phys D14 (2005) 171; B Mashhoon, Phys Rev D90 (2014) 124031

[12] Misner, Thorne K and Wheeler J A, Gravitation, Freeman (1972)

[13] Synge J L, Relativity: the special theory, North-Holland (1965)

[14] Walker A G, Proc Roy Soc Edinburgh 52 (1932) 345

[15] Bini D and Mashoon B, Phys Rev D 91 (2015) 084026

[16] Marzlin K-P, Gen Relativ Gravit 26 (1994) 619; Marzlin K-P, Phys Rev D50 (1994) 888 
[17] Llosa J, "Einstein's accelerated reference systems and Fermi-Walker coordinates", arxiv:1706.09656[physics.class-ph]

[18] Ni W-T and Zimmermann M, Phys Rev D 17(1978) 1473

[19] Gourgoulhon E, Special relativity in general frames, Springer 2013

[20] Bimonte G, Calloni E, Esposito G and Rosa L, Phys Rev D74 (2006) 085011

[21] Korsbakken J I and Leinaas J M, Phys Rev D70 (2004) 084016

[22] Parker L, Phys Rev D22 (1980) 1922

[23] ParkerL, Phys Rev D25 (1982) 3180

[24] Chicone C and Mashhoon B, Ann Phys (Leipzig) 16 (2007) 811

[25] Collas P and Klein D, Gen Relativ Gravit 39 (2007) 737

[26] Ishii M, Phys Rev D71 (2005) 044017

[27] Friedman Y and Scarr T, Gen Relativ Gravit 47 (2015) 121

[28] Mashhoon B and Muench U, Ann Phys (Leipzig) 11 (2002) 532

[29] Bel L, "Born's group and generalized isometries", in Relativity in General. Proceedings of the Relativity Meeting'93, J Diaz and M Lorente eds., Editions Frontières (1994)

[30] Hicks N, Notes on differential geometry, Van Nostrand (1965)

[31] Einstein A, Phys Zeitschr 14 (1913) 1249 